\section{Flow dynamics study of catalyst powder in catalytic cracking unit for troubleshooting}

\begin{abstract}
Gamma scanning and radiotracer applications are very effective and inexpensive tools to understand and optimize the process as well as troubleshoot the various types of problems in many chemical, petrochemical industries and refineries. These techniques are non-invasive; hence, the problems can be pinpointed online, which leads to reduce the downtime, schedule the shutdown and maintenance of the plant equipment, rendering huge economic benefits. In a leading refinery of India, the catalytic cracking unit (CCU) was malfunctioning. It was suspected by the refinery engineers that the catalyst powder was being carried over to the fractionator, which could have led to erosion of the fractionator column internals resulting in their rupture, and consequentially, to the fire hazard. To understand the flow behaviour of the catalyst powder and to ensure the mechanical integrity, catalyst accumulation and choking, both radiotracer study and gamma scanning of the CCU reactor was carried out. The reactor consists of a riser, three primary cyclones and three secondary cyclones. Gamma scanning of the reactor was carried out with the help of an automatic gamma scanner using $1.8 \mathrm{GBq}$ of Co-60 sealed source. Results showed that the catalyst powder was accumulated in one of the secondary cyclones and uneven density distribution was observed in another secondary cyclone. The radiotracer study was carried out using the irradiated catalyst powder as a radiotracer, which contains $0.9 \mathrm{GBq}$ of $\mathrm{Na}-24$. The radiotracer was injected in the reactor through the specially fabricated injection system. Radiation measurement was done using the thermally insulated and collimated $\mathrm{NaI}(\mathrm{Tl})$ scintillation detectors located at various strategic locations coupled to a multi-detector data acquisition system. The data were mathematically analysed. It was confirmed that the catalyst powder was accumulated in one of the secondary cyclones with no flow downwards. This resulted in excess powder available to travel along with hydrocarbon towards fractionator. Since the quantity of powder released through the hydrocarbon outlet of CCU was higher than the designed value, the catalyst powder was observed in various zones of the fractionator. Mathematical modelling of the radiotracer data obtained at various locations corroborated the scanning results; also, the flow pattern was obtained. Partially blocked secondary cyclone showed plug flow with recirculation; normal working cyclone had plug flow behaviour and the vortex breaker showed parallel flow.
\end{abstract}

Keywords: catalytic cracking unit $\bullet$ cyclones $\bullet$ fractionator $\bullet$ radiotracer $\bullet$ plug flow

V. Yelgaonkar ${ }^{\bowtie}$, G. Agrahari, D. Vikrant, R. Prasanna, B. K. Pathak

Board of Radiation \& Isotope Technology,

Department of Atomic Energy,

Government of India,

Vashi Complex,

Navi Mumbai, India,

E-mail: yelgaonkar@britatom.gov.in

Received: 19 April 2017

Accepted: 15 November 2017

\section{Introduction}

Catalytic cracking is one of the most important processes in the petroleum refineries to convert the high molecular weight hydrocarbons to low molecular weight valuable products. The preheated high-boiling petroleum feedstock (at about 315 to $430^{\circ} \mathrm{C}$ ) consisting of long-chain hydrocarbon molecules is combined with recycle slurry oil from the bottom of the distillation column and injected into the catalyst riser, where it is vapourized and cracked into smaller molecules of vapour by contact and mixing with the very hot powdered catalyst from the regenerator. All the cracking reactions take place in the catalyst riser within a period of 2-4 seconds. The mixture of hydrocarbon vapours and catalyst flows upward to enter the reactor at a temperature of about $535^{\circ} \mathrm{C}$ and a pressure of about 1.72 bar. Malfunctioning of 
these units is a common phenomenon in the refineries. In one of the leading refineries of India, the reactor of CCU was suspected to be malfunctioning. The catalyst powder entered the fractionator column after cracking, which is an undesirable phenomenon. The reactor consists of a riser, three primary cyclones (RC1, RC2 and RC3) and three secondary cyclones (SC1, SC2 and SC3). To ascertain the cause of malfunctioning through radioisotope techniques, it was decided to carry out gamma scanning for secondary cyclones. Three scan lines were identified to understand the condition of secondary cyclones. Further, to understand the behaviour of catalyst movement inside the reactor, the radiotracer study was carried out. Major advantage of the gamma scanning and radiotracer techniques is its utility without disturbing the process, that is, it is carried out online. Conventional methods of troubleshooting like pressure drop studies, viscosity measurements, sampling and so on can provide a rough idea about the problem in the system but they cannot pinpoint the problem area. Also, these techniques are either offline measurement techniques or cannot be applied in industrial systems for online measurement due to their complexity and the harsh conditions. Whereas, gamma scanning and radiotracer studies are used worldwide for effective trouble shooting and to arrive at exact problem location due to a number of advantages such as high detection sensitivity, in situ detection, physico-chemical compatibility, limited memory effect, unambiguous detection and ability to obtain results quickly. This minimizes the expensive down time of the large capacity petroleum refineries.

\section{Gamma scanning}

Performance of an industrial process column and vessels depends upon its mechanical design and chemical process optimization. Any malfunctioning of the columns, depending on their size, can cause huge production losses resulting in high revenue losses. Gamma scanning technique is a very useful on-line troubleshooting tool to identify the problem as well as to assess the column/vessel hardware and its general health for chemical and petrochemical industries. Gamma scanning provides a non-destructive and cost-effective way of analysing problems of process columns/vessels. Scan data is useful for scheduling shutdowns, estimating turnarounds, carrying out periodic maintenance and process optimization.

The transmission of $\gamma$ radiation through any material is governed by the exponential equation:

$$
I=I_{o} e^{-\mu x}
$$

where: $I$ - intensity of radiation transmitted through a material of thickness $\mathrm{X}, \mathrm{cm} ; I_{o}$ - intensity of radiation from the $\gamma$-ray source reaching the detector in the absence of the material; $\mu$ - linear absorption coefficient, $\mathrm{cm}^{-1}$.

A collimated source and detector system is positioned in the same horizontal plane, either across the diameter or selected chords with the help of pul-

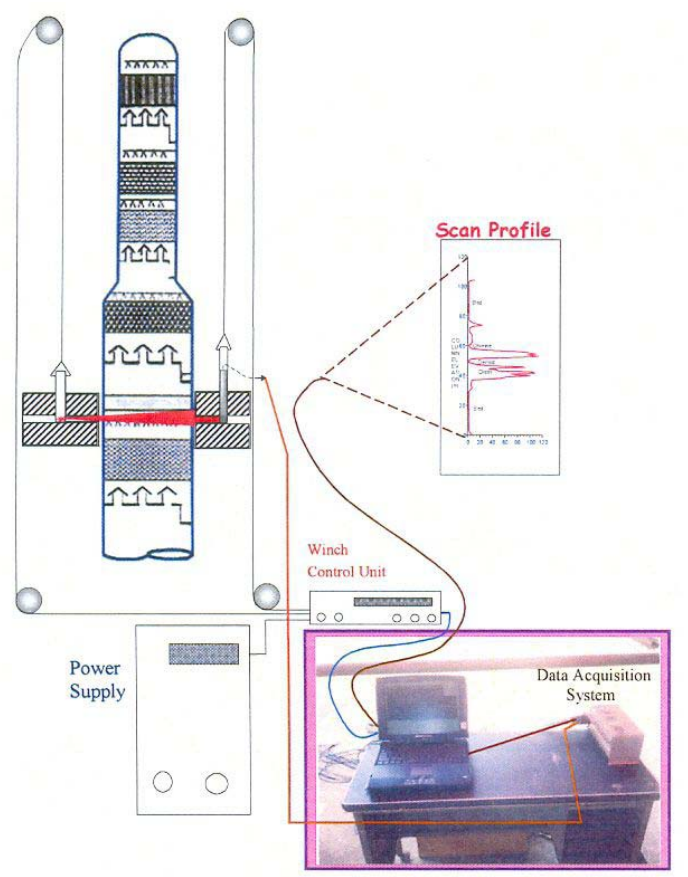

Fig. 1. General setup of gamma scanning process.

leys and wire ropes connected to a winch system as shown in Fig. 1. After assessing the situation, a detailed study was carried out to select proper chords depending upon the orientation of cyclones. Both the source and detector are moved synchronously along the length of the reactor and the radiation intensity is recorded at desired elevations by using nucleonic scalar rate meter. The data thus generated are plotted against the reactor elevation and interpreted with reference to the internal loading and hardware configuration of the reactor to derive the necessary information.

\section{Radiotracer study}

A radiotracer is injected in the system and then measured at the outlet of the system. A radiotracer has the same physico-chemical properties as the substance or particles it represents, and can be detected by an analytical instrument without disturbing the system.

The theory of radiotracer studies allows the treatment of systems with many connections to their environments, with multidirectional flow in the connections, with time dependent flow rates and even with time dependent system boundaries. The age of particles when they enter the system is equal to zero and equal to the residence time when they finally leave the system. For this purpose, the molecules that enter during a given interval of time are marked, and they are counted as a function of time in the outlet streams. With the help of a tracer, a given signal is imposed at the inlet of the reactor and the system response is collected at the outlet, as a function of time as shown in Fig. 2.

Supposing that the injection of the tracer is made instantaneously at $t=0$ (Dirac's impulse), if $C_{\text {out }}(t)$ is the curve representing the concentration of tracer 


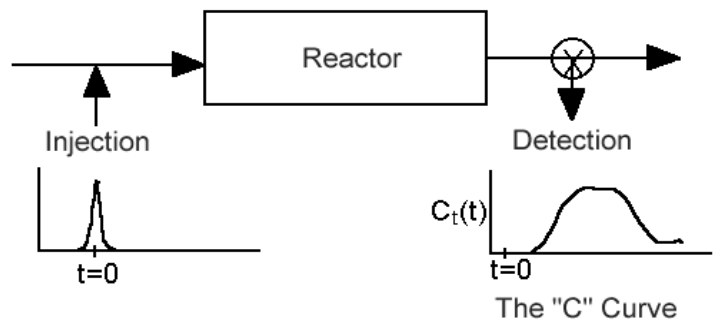

Fig. 2. System response for a tracer input.

at the outlet of the reactor, the fraction of molecules that remain within the interval of time $t$, is better known as residence time distribution (RTD) $E(t)$ determined by:

$$
E(t)=\frac{C_{\text {out }}(t)}{\int_{0}^{\infty} C_{\text {out }}(t) d t}
$$

As long as the tracer has exactly the same flow properties as the other particles and thus follows the same paths, the tracer concentration $C_{\text {out }}(t)$ registered at the outlet in time $t$ is directly proportional to the value $E(t)$ (more precisely: $E(t)$ is proportional to the number of particles detected per unit time). $E(t)$ is, by definition, the impulse response (or RTD) of the system as depicted in Fig. 2. $E(t)$ is a convenient variable to present the RTD, since it can be obtained by dividing the concentration $C_{\text {out }}(t)$ by the total area under the curve. An $E(t)$ curve is shown in Fig. 3.

The first moment specifies the position of the centroid, the mean of the residence time.

$$
\begin{gathered}
M_{1}=\bar{t}=\int_{0}^{\infty} t E(t) d t \\
\bar{t}=\frac{\int_{0}^{\infty} t C_{\text {out }}(t) d t}{\int_{0}^{\infty} C_{\text {out }}(t) d t}
\end{gathered}
$$

The physical sense of the mean residence time can be expressed in the relation:

$$
\bar{t}=\frac{\mathrm{V}}{\mathrm{v}}
$$

where $\mathrm{V}$ is the system volume and $v$ is the volumetric flow rate.

The second moment, the variance of distribution function is given by

$$
\bar{M}_{2}=\sigma^{2}=\int_{0}^{\infty}(t-\bar{t})^{2} E(t) d t
$$

or

$$
\sigma^{2}=\frac{\int_{0}^{\infty}(t-\bar{t})^{2} C_{\text {out }}(t) d t}{\int_{0}^{\infty} C_{\text {out }}(t) d t}=\frac{\int_{0}^{\infty} t^{2} C_{\text {out }}(t) d t}{\int_{0}^{\infty} C_{\text {out }}(t) d t}-\bar{t}^{2}
$$

To compare the RTD's of different systems, their dimensionless forms are often used. The dimensionless residence time distribution is then given by:

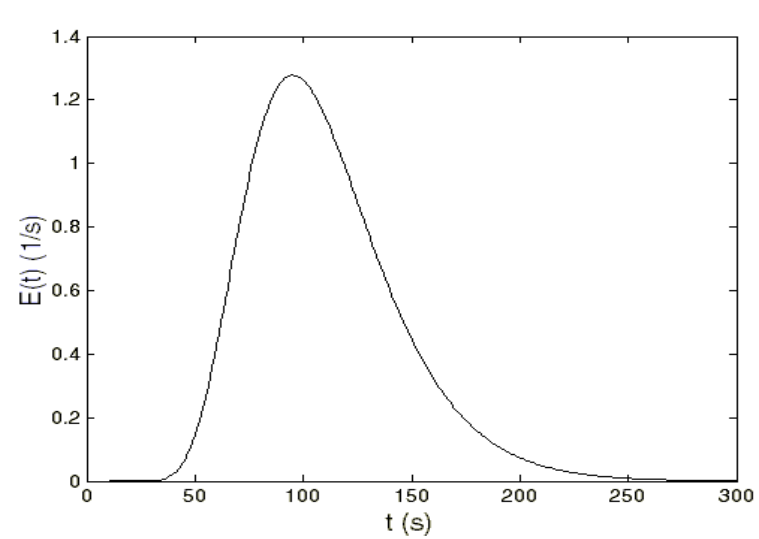

Fig. 3. Residence time distribution curve $E(t)$.

$$
E(\theta)=\bar{t} E(t)
$$

where the dimensionless time $\theta$ is the ratio of real time $t$ and mean residence time $\bar{t}$.

$$
\theta=\frac{t}{\bar{t}}
$$

The response of a flow system to a stimulus must be measured under steady state conditions in which the volumetric flow rate, $v$ and the volume $\mathrm{V}$ of the system are kept constant. $E(t)$ values are obtained directly by normalizing the values of the measured response only in cases when the stimulus function may be considered as an ideal impulse, that is, when the duration of the stimulus is less than $3 \%$ of the mean residence time. If this condition is not fulfiled, the input stimulus must also be recorded and normalized. This normalization is performed by dividing the measured concentration by the respective area under the concentration-time curve. Thus, on normalization we have input and output signals as:

and

$$
X(t)=\frac{C_{i n}(t)}{\int_{0}^{\infty} C_{i n}(t) d t}
$$

$$
Y(t)=\frac{C_{\text {out }}(t)}{\int_{0}^{\infty} C_{\text {out }}(t) d t}
$$

\section{Modelling of the RTD data}

According to the shape of RTD function and the character of the apparatus, it is possible to suggest a mathematical model, that is, a description of responses by means of a system of differential and/or algebraic equations. In this study, THYNRTD2 software was used to analyse the data and describe the appropriate models. It is possible to describe the models with time variable flow rates, variable volumes of units, and so on. The solution of this system is usually performed by numerical integration in the time domain (using Runge-Kutta or Euler method) and the estimation of model parameters is based upon the least squares method, that is, upon minimizing the sum of squares of differences between the mea- 
sured and computed responses. This is generally a non-linear regression problem that can be solved, for example, by the Marquardt-Levenberg method adapted for models defined as a set of ordinary differential equations. A model of the analysed system is to be described in the RTD2 program by a set of ordinary differential equations or algebraic expressions. Consider as an example the system of two serially connected and ideally mixed vessels with internal volumes V1 and V2. Time courses of concentration in individual vessels $\mathrm{C} 1(t), \mathrm{C} 2(t)$ corresponding to a general stimulus function $x(t)$ are described by the following equations:

$$
\begin{aligned}
\mathrm{V} 1 \frac{d c_{1}}{d t} & =Q\left(x-x_{1}\right) \\
\mathrm{V} 2 \frac{d c_{2}}{d t} & =Q\left(c_{1}-c_{2}\right)
\end{aligned}
$$

together with initial conditions for concentrations and with appropriate set of parameters. In this small example, we might have

$$
\begin{aligned}
& \mathrm{P} 1=\mathrm{V} 1 / Q \\
& \mathrm{P} 2=\mathrm{V} 2 / Q
\end{aligned}
$$

Because all the model variables and parameters (e.g., volumes $\mathrm{V}$ and flow rate $Q$ ) may depend upon time, the system with variable flow rates or volumes (generally systems with time dependent flow structure) can be easily analysed. Time delay (plug flow regions), recycle loops, multiple inlet/outlet streams can also be incorporated into the model definition.

\section{Experimental}

\section{Catalytic cracking unit}

The feed system is designed to ensure a constant feed rate to the unit - hot waxy distillate at a temperature of $230^{\circ} \mathrm{C}$ from feed preparation unit or high vacuum unit, long residue (LR) from the crude distillation unit is pumped as feed in the catalytic cracking unit (CCU). In the reactor lift pot, the feed from charge heater at a temperature of $290-360^{\circ} \mathrm{C}$ is mixed with stream of hot catalyst coming from the regenerator, which is at a sufficiently high temperature of about $680^{\circ} \mathrm{C}$ to vapourize the feed. The catalyst is a very fine powder of crystalline material (zeolites), which has the property that on mixing with gas/vapours it behaves like a fluid, and therefore, flows. The catalyst vapourized feed mixture flows upward through reactor riser in which the cracking reaction taken place. The cracked vapours and catalyst are separated in three two stage rough-cut cyclones. The separated hydrocarbons from cyclones go to fractionator where the vapours are separated into different products depending on their boiling point range. As a result of the cracking reactions, some heavy hydrocarbon material called coke is deposited on the catalyst, which deactivates the catalyst. Also, the cracked heavy hydrocarbons get associated with catalyst surface, which require to be stripped before catalyst goes back to regenerator. The catalyst from the cyclone dip legs flows counter current downwards through the annular baffles for pre-stripping into the pre-stripper bed (as dense phase in the reactor lower section). Five (5) $\mathrm{kg} / \mathrm{cm}^{2}$ steam is used for pre-stripping and the flow rate is controlled by the control valve. The stripped catalyst or the "spent catalyst" flows to the regenerator, where the coke is burnt off to restore catalyst activity. The air required for combustion of coke is introduced in regenerator through main air blower. A continuous flow of regenerated catalyst is withdrawn from the regenerator and fed to the bottom of the reactor riser. The regenerator slide valve controls the catalyst circulation rate.

\section{Gamma scanning}

As seen from Fig. 1, general setup of the gamma scanning equipment, the source and detector collimator is hanged from the top of the reactor. The arrangement of pulleys is made at pre-determined suitable locations. From the drawings, details of internal mechanical hardware of the reactor were known. The process/operations engineer marks the points at which the pulleys can be affixed as well as lines are marked, which should be followed by the wire ropes of source and detector. Orientation of the scan lines were identified as per Fig. 4. 1.8 GBq of Co-60 (half-life -5.27 years, energy -1.17 and $1.33 \mathrm{MeV}$ ) sealed source was used, which was collimated using a specially designed tungsten collimator. Collimated BGO detector was used to record the counts from co-planar collimated gamma radiation source. Both source and detector collimators have $65 \mathrm{~mm}$ outer diameter that required passing between the platform and body of the column. Wherever necessary, at some locations, holes were made on the platforms or gratings were removed to facilitate the smooth passage of the collimators. Automatic gamma scanning system consisting of source operator and detector operator was installed at the fourth level of CCU reactor. The source and detector operators were controlled through a remote-control unit and data of counts

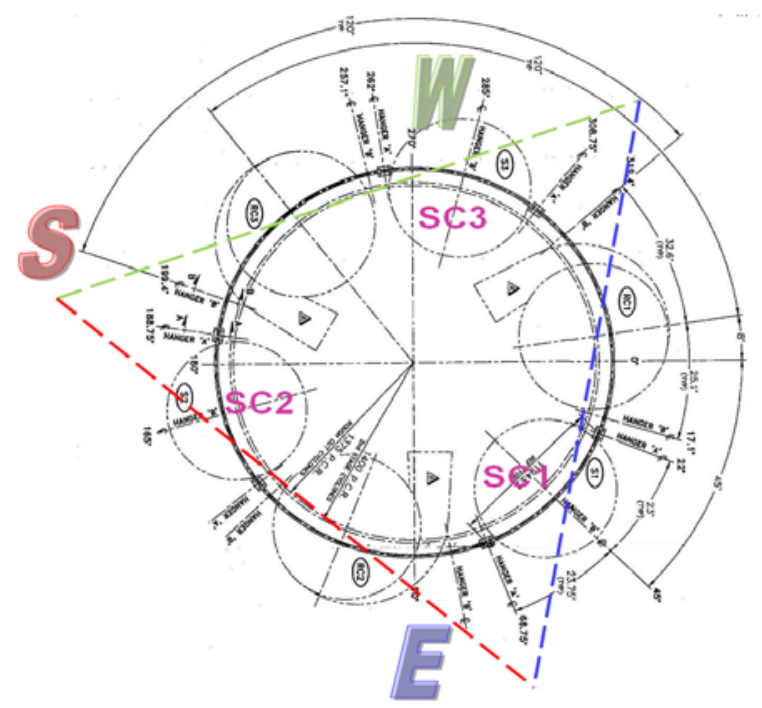

Fig. 4. Scan line orientation for gamma scanning. 


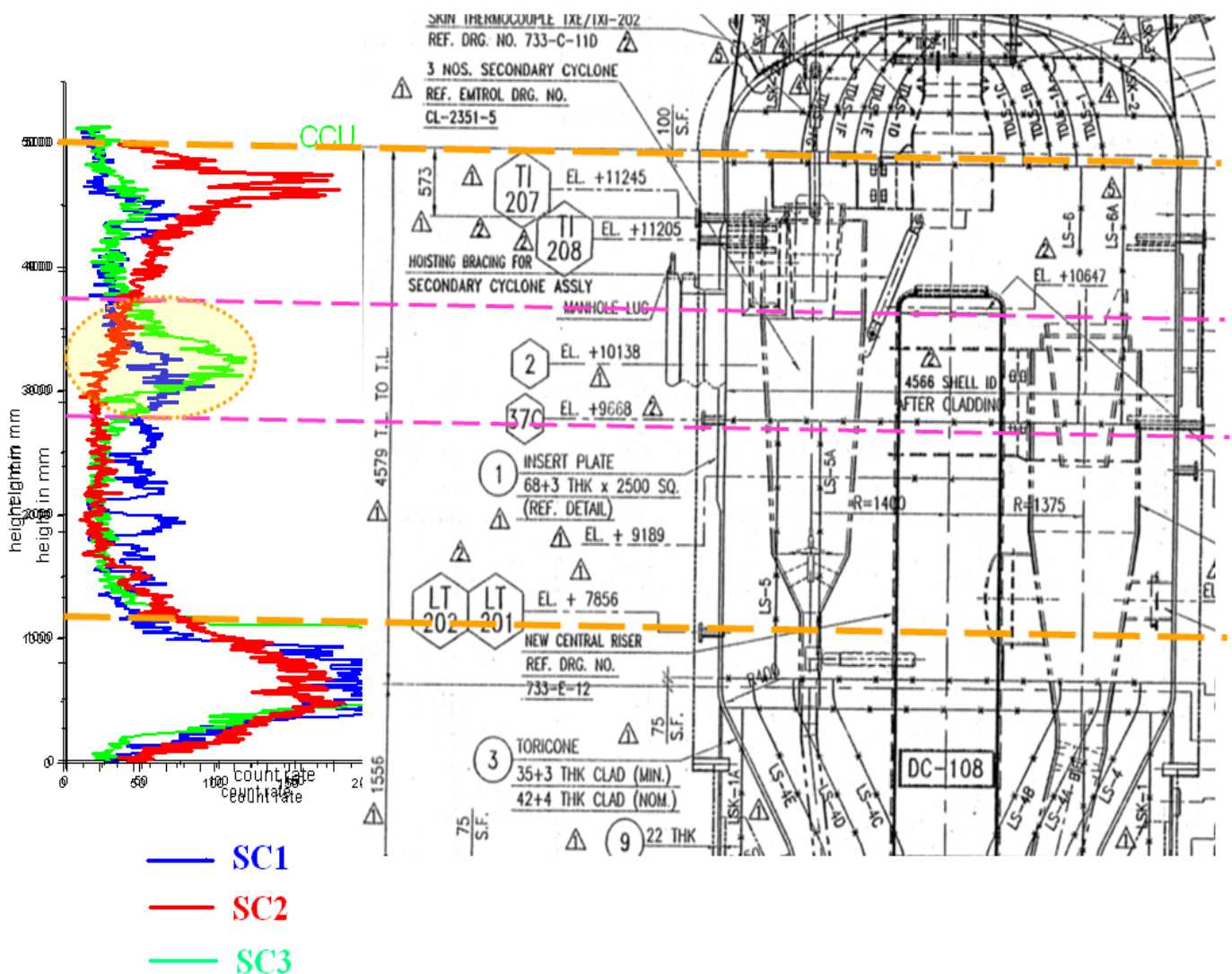

Fig. 5. Count rate profile with respect to secondary cyclones.

per 3 seconds with respect to the elevation at step height of $50 \mathrm{~mm}$ were obtained and recorded in the laptop. The count rate profile obtained has been shown in Fig. 5.

\section{Radiotracer investigation}

The radiotracer study was carried out using $3 \mathrm{gm}$ irradiated catalyst powder as a radiotracer, which

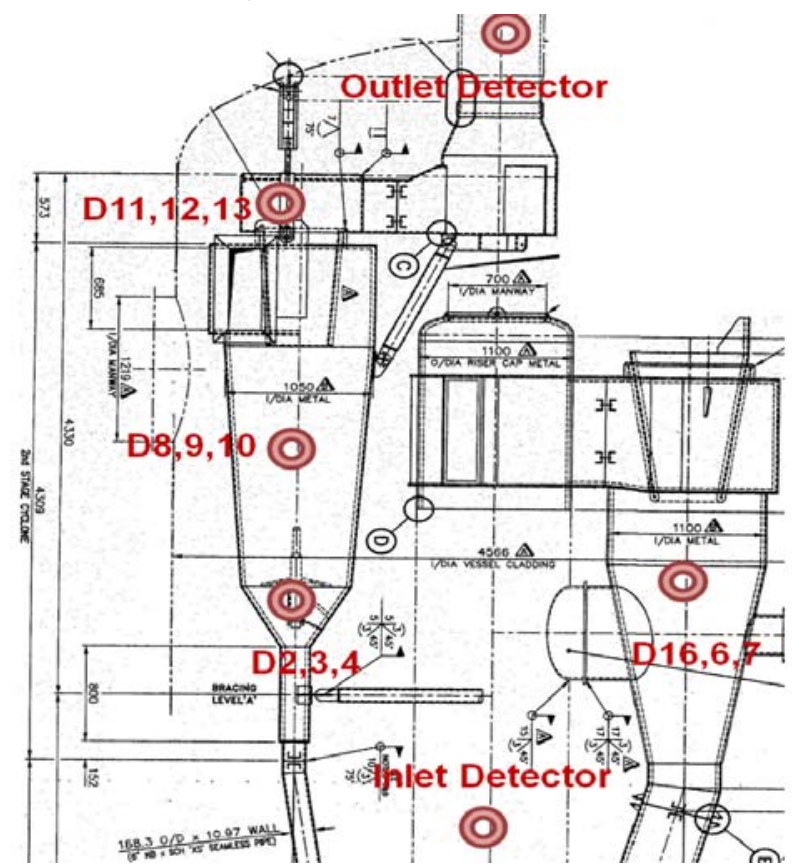

Fig. 6. Detector positioning at various locations. majorly contains $0.9 \mathrm{GBq}$ of $\mathrm{Na}-24$ (half-life $-15 \mathrm{~h}$, energy $-1.37,2.15 \mathrm{MeV}$ ). The tracer was instantaneously injected into the reactor through a nozzle in the lift pot using a specially fabricated injection system. Three injections were carried out in the reactor. The handling of radioisotope was carried out through specially designed tools to ensure radiation safety at the site. The tracer was monitored at 14 different strategically selected locations using collimated 2 inch $\times 2$ inch $\mathrm{NaI}(\mathrm{Tl})$ scintillation detectors, as shown in Fig. 6. The details have been given in Table 1. All the detectors were calibrated prior to the experiment in the laboratory to give identical responses. All the detectors were connected to a computer controlled multi input data acquisition system (MIDAS) to record the tracer concentra-

Table 1. Detector locations

\begin{tabular}{rcc}
\hline $\begin{array}{c}\text { Serial } \\
\text { no. }\end{array}$ & $\begin{array}{c}\text { Detector } \\
\text { no. }\end{array}$ & Location detail \\
\hline 1 & 2 & SC1 (vortex breaker) \\
2 & 3 & SC3 (vortex breaker) \\
3 & 4 & SC2 (vortex breaker) \\
4 & 6 & RC2 \\
5 & 7 & RC1 \\
6 & 8 & SC3 (secondary body) \\
7 & 9 & SC1 (secondary body) \\
8 & 10 & SC2 (secondary body) \\
9 & 11 & SC2 (outlet of plenum chamber) \\
10 & 12 & SC3 (outlet of plenum chamber) \\
11 & 13 & SC4 (outlet of plenum chamber) \\
12 & 14 & Outlet \\
13 & 15 & Riser \\
14 & 16 & RC3 \\
\hline
\end{tabular}



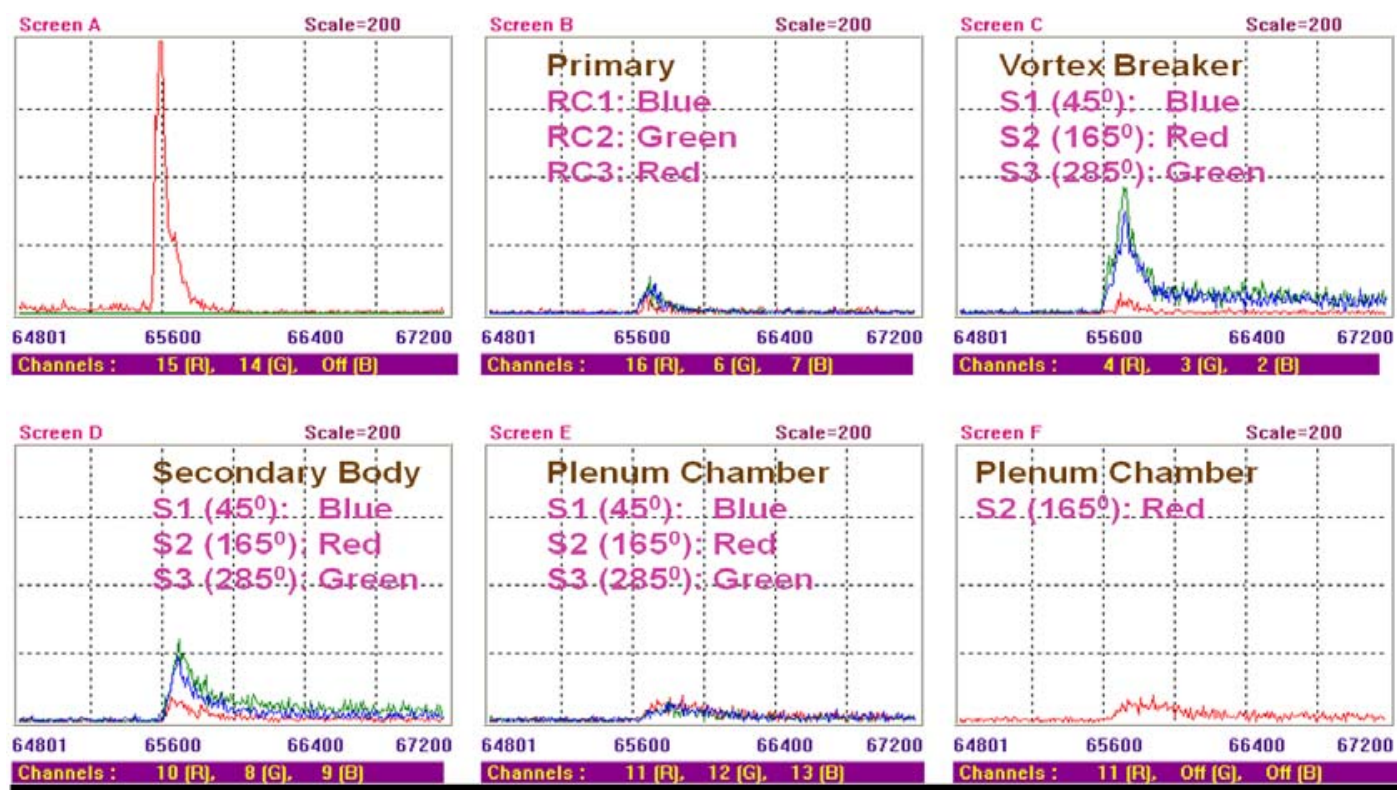

Fig. 7. Detector response curves at various locations.

Table 2. Analysis of peaks at secondary cyclone body location

\begin{tabular}{lcccc}
\hline Serial no. & $\begin{array}{c}\text { Secondary cyclone } \\
\text { [angle] }\end{array}$ & $\begin{array}{c}\text { Area under tracer } \\
\text { curve }\end{array}$ & $\begin{array}{c}\text { Percentage of powder } \\
\text { travelled through }\end{array}$ & $\begin{array}{c}\text { Mean residence time } \\
{[\mathrm{s}]}\end{array}$ \\
\hline SC1 & 45 & 17532 & 35 & 1.58 \\
SC2 & 165 & 6483 & 13 & 1.56 \\
SC3 & 285 & 26841 & 52 & 2.18 \\
\hline
\end{tabular}

Table 3. Analysis of peaks at secondary cyclone vortex breaker location

\begin{tabular}{|c|c|c|c|c|}
\hline Serial no. & $\begin{array}{l}\text { Secondary cyclone } \\
\text { [angle] }\end{array}$ & $\begin{array}{l}\text { Area under tracer } \\
\text { curve }\end{array}$ & $\begin{array}{l}\text { Percentage of powder } \\
\text { travelled through }\end{array}$ & $\begin{array}{l}\text { Mean residence time } \\
{[\mathrm{s}]}\end{array}$ \\
\hline$\overline{\text { SC1 }}$ & 45 & 29799 & 42 & 2.34 \\
\hline $\mathrm{SC} 2$ & 165 & 2517 & 4 & 1.94 \\
\hline SC3 & 285 & 38593 & 54 & 2.28 \\
\hline
\end{tabular}

Table 4. Analysis of peaks at outlet of plenum chamber location

\begin{tabular}{lcccc}
\hline Serial no. & $\begin{array}{c}\text { Secondary cyclone } \\
\text { [angle] }\end{array}$ & $\begin{array}{c}\text { Area under tracer } \\
\text { curve }\end{array}$ & $\begin{array}{c}\text { Percentage of powder } \\
\text { travelled through }\end{array}$ & $\begin{array}{c}\text { Mean residence time } \\
{[\mathrm{s}]}\end{array}$ \\
\hline SC1 & 45 & 80164 & 17.5 & 77.5 \\
SC2 & 165 & 333205 & 73.0 & 192 \\
SC3 & 285 & 43712 & 9.5 & 6.18 \\
\hline
\end{tabular}

tion at pre-defined time intervals of every $20 \mathrm{~ms}$. The acquired concentration vs. time data were recorded at different locations, corrected for background radiation and plotted against time, as shown in Fig. 7. The data were analysed using Thyn's RTD software.

\section{Results and analysis}

\section{Results obtained from gamma scanning}

As per the count rate profiles shown in Fig. 5, following interpretations could be made:

- In secondary cyclone SC1, uneven density distribution was observed indicating disturbances in catalyst powder flow.

- Secondary cyclone SC2 was filled with powder.
Secondary cyclone SC3 showed normal catalyst flow.

\section{Results obtained from radiotracer investigations}

The detectors' positioning was according to Table 1 and their corresponding curves were shown in Fig. 7. The areas of peaks were determined for detector locations of secondary cyclone body (Table 2), secondary cyclone vortex breaker (Table 3) and outlet of plenum chamber (Table 4). Depending upon the area under the tracer curves, the percentage of tracer movement at each point was determined. The mean residence time of the tracer in the system at that location was also determined. Following interpretations could be made: 


\section{Secondary cyclone SC1}

- Secondary cyclone CS1 showed partial filling of catalyst powder. The quantity of catalyst powder received was $35 \%$.

- The vortex breaker received $42 \%$ catalyst powder.

- Release of radiotracer catalyst powder through the outlet of SC1 to plenum chamber was $17.5 \%$.

- It appeared that SC1 also contributes slightly higher catalyst flow towards the fractionator.

\section{Secondary cyclone SC2}

- Since secondary cyclone SC2 was filled with powder, the quantity of radiotracer catalyst powder received was minimum (13\%).

- The same trend was reflected in vortex breaker where only $4 \%$ of the powder escaped.

- Outlet of SC2 to plenum chamber slowly released the radiotracer catalyst powder (in $192 \mathrm{~s}$ ). Quantity of powder released through this outlet was very high (73\%) which could be responsible for catalyst powder carrying over towards fractionator.

\section{Secondary cyclone SC3}

- Secondary cyclone SC3 received maximum quantity of catalyst powder (52\%).

- Vortex breaker of this cyclone released $54 \%$ of catalyst powder, indicating good working of the cyclone.

- Outlet of SC3 to plenum chamber released the least quantity of powder (9.5\%).

Similar trends were observed in second and third injections. Also, these results verified the results obtained by gamma scanning experiments.

\section{Modelling of the radiotracer data}

The data recorded for the secondary cyclones and vortex breaker was modelled to obtain the flow pattern. Following results were obtained:

- Secondary cyclone SC3 showed plug flow behaviour.

- Secondary cyclone SC1 showed plug flow with recirculation.

- The vortex breaker showed the parallel flow behaviour.

Acknowledgment. Authors gratefully acknowledge the encouragement from Chief Executive, BRIT for continuous encouragement during the study and the refinery engineers for their untiring help to perform the study.

\section{References}

1. Charlton, J. S. (1986). Radioisotope techniques for problem solving in industrial process plants. Glasgow: Leonard Hill.

2. International Atomic Energy Agency. (1990). Guidebook on radioisotope tracers in industry. Vienna: IAEA. (Technical Report Series no. 316).

3. Zitny, R., \& Thyn, J. (1996). Programs for residence time distribution analysis, RTD software manuals for data analysis of radiotracer experiments. Vienna: International Atomic Energy Agency. 